\title{
Modeling of MIMO-OFDM Channel with STBC and Directivity to Combat Fading and Co-Channel Interference
}

\author{
Ziyad Khalaf Farej, Ahmed T. Al-Najafi \\ Technical Engineering College, Northern Technical University, Mosul, Iraq \\ Email: drziyad.farej@ntu.edu.iq, master.aft@gmail.com
}

How to cite this paper: Farej, Z.K. and Al-Najafi, A.T. (2020) Modeling of MIMOOFDM Channel with STBC and Directivity to Combat Fading and Co-Channel Interference. Open Access Library Journal, 7: e6809.

https://doi.org/10.4236/oalib.1106809

Received: September 10, 2020

Accepted: November 14, 2020

Published: November 17, 2020

Copyright $\odot 2020$ by author(s) and Open Access Library Inc.

This work is licensed under the Creative Commons Attribution International License (CC BY 4.0).

http://creativecommons.org/licenses/by/4.0/

\begin{abstract}
A wireless communication system for cellular technology is currently considered a very important topic for mobile phone. The design of such systems needs to include their multipath and fading main problems. In this paper, a MIMO-OFDM channel is designed and modeled to combat such problems, as well as STBS and directivity are also involved in this design to increase system reliability and enhance BER performance. The modeling and simulation process of the designed systems are carried out using Matlab software (ver. R2019a). Without directivity, simulation process shows that including STBC results on average a gain of $\approx 1.5 \mathrm{~dB}$ for different spatial stream. With respect to the no directivity $(2 \times 1$ case), when directivity of $4 \times 1$ and $8 \times 1$ spatial streams are introduced (at fixed $15 \mathrm{~dB}$ S/N), the BER improvement values are $85 \%$ and $95 \%$ for $5 \mathrm{Km}$ (cluster size $\mathrm{k}=4$ ) and $9.5 \mathrm{Km}$ (cluster size $\mathrm{k}=7$ ) co-channels interferers position for BS respectively.
\end{abstract}

\section{Subject Areas}

Simulation/Analytical Evaluation of Communication Systems

\section{Keywords}

MIMO, STBC, Beamforming, Cellular, Interferers, Co-Channels

\section{Introduction}

Multiple Input Multiple Output (MIMO) communication is a collection of signal processing techniques that have been developed to enhance the performance of wireless communication systems, using multiple antennas at the transmitter, receiver, or both. MIMO techniques improve communications performance by 
either combating or exploiting multipath scattering in the communications channel between a transmitter and receiver. The two most important MIMO techniques are spatial diversity which improves reliability by combating fading, and spatial multiplexing, which is normally used for maximizing throughput, through the process of feeding independent data into each antenna, with all antennas transmitting at the same frequency. Through its different multiple-antenna technique, spatial multiplexing creates multiple channels with independent streams to increase the overall data capacity, while in spatial diversity multiple copies of the same signal are transmitted via multiple antennas to increase the chance of properly receiving the signal and improve reliability through the overall signal to noise ratio enhancement, and as a result decreasing in the system BER and improvement in the network efficiency.

In wireless communication channel, the fading can be modeled as Rayleigh distributed when there is no line of sight (LoS) component present in the received signal, as shown in Figure 1. LoS signal may also be characterized by a component that contains considerably higher power than collective power of all the remaining components [1].

To prevent and combat the Rayleigh fading, space time block code (STBC) is used. The STBC is applied through the spatial diversity technique, which is considered in this paper. In this technique, the information bits are normally encoded and modulated using conventional error correction coding and modulation techniques before applying space time block coding. At the receiver, STB decoding is performed followed by demodulation and error decoding, as shown in Figure 2.

In addition, at the base station, the advanced MIMO system and through its multi-antenna technologies support beamforming to improve the communication range (or data rate) and also beam steering (which means dynamically creating and pointing beam in the desired direction) technology to avoid adjacent or co-channel interference (especially in cellular system). The co-channel interference for different cluster sizes $(k=4, k=7)$ is shown in Figure 3.

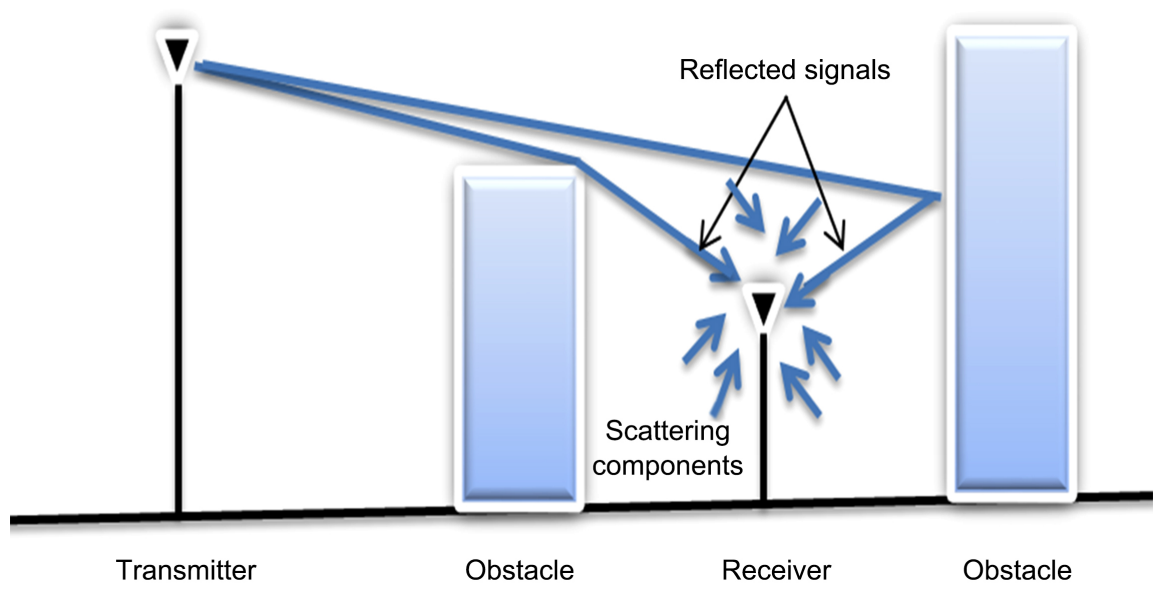

Figure 1. Shows multipath of wireless channel. 


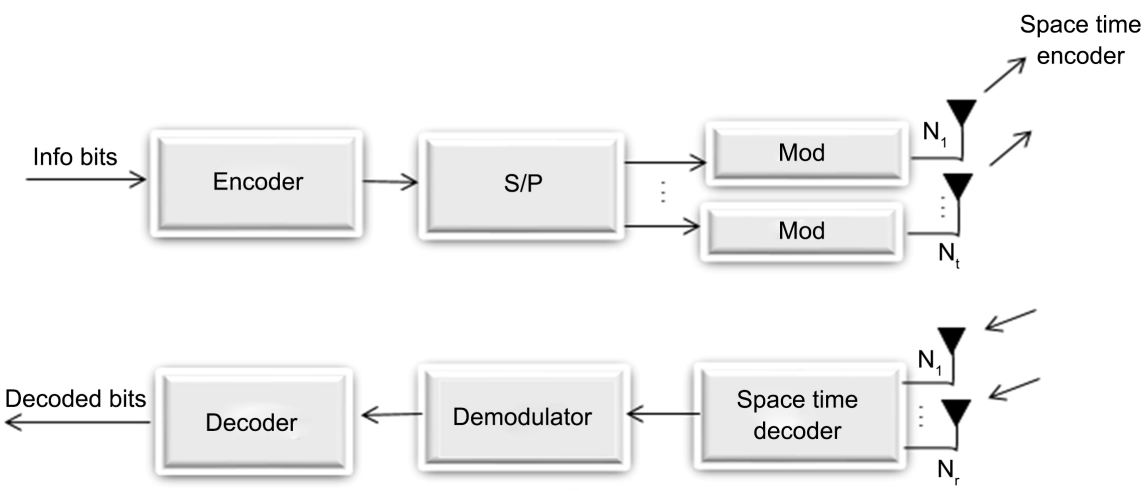

Figure 2. MIMO system for spatial diversity.

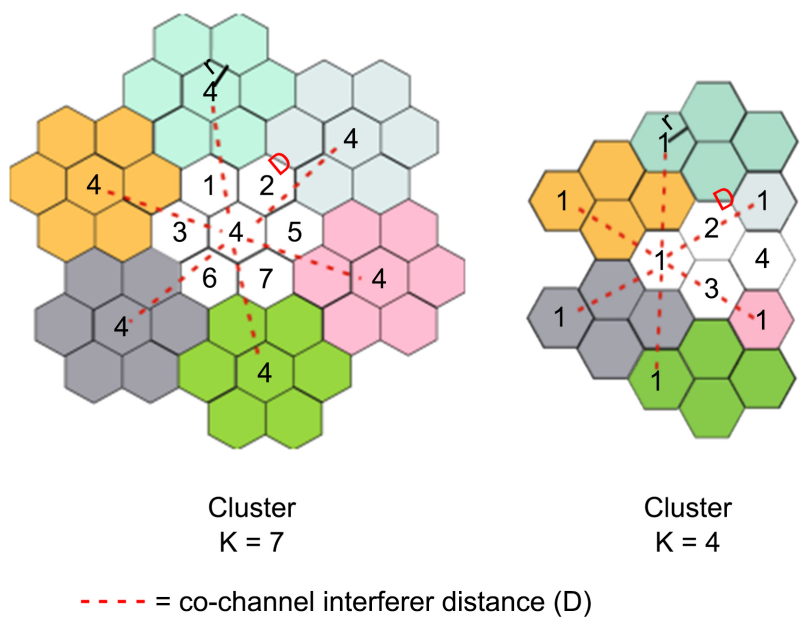

Figure 3. Shows cells 4 and 1 co-channel interference for clusters $k=7$ and $k=4$ respectively.

By varying the relative phase and amplitudes of the applied signals to the individual ( $4 \mathrm{G}$ or $5 \mathrm{G}$ ) base station elements, the MIMO antenna system can steer and shape a beam in the desired direction. Figure 4 shows the initial access technique where the base station uses beam sweeping to transmit multiple beams to identify the strongest beam and establish a communication link [2].

\section{Related Works}

The performance of wireless communication system is highly degraded by multipath problem. However the effect of this problem, which is addressed in our proposed communication model, is reduced through OFDM technique which uses a set of subcarriers to satisfy the required OFDM symbol length for certain (maximum) multipath delay. The performance of the MIMO-OFDM channel is analyzed and investigated by many researches [3] [4] [5]. Their analyses and simulation results clarified that the bit error rate and channel efficiency can be optimized through different scenarios, spatial streams and simulation parameters configuration. Jeiad studied, the effect of STBC on the multipath fading channel [6], their simulation results show additional enhancement in the BER when the 
Initial access and beam management

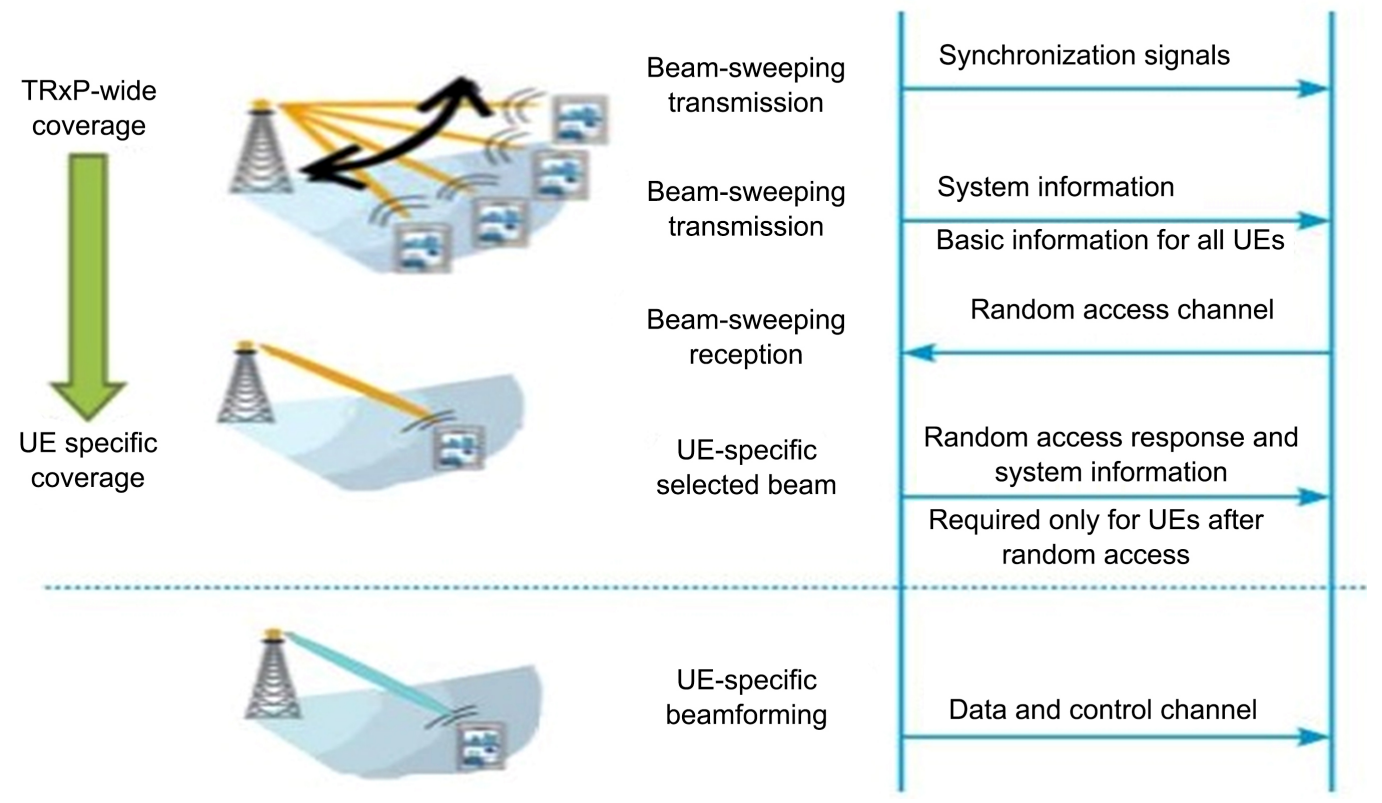

Figure 4. Illustrates process and initial access of MIMO system for 4G and 5G basestation.

STBC is combined with interleaving and Reed-Solomon coding. Capacity improvement in the wireless communication system is considered by [7]. Through the use of multiple antennas at transmitter and receiver side (MIMO) and space time coding code (STBC) the capacity of this system is enhanced and the BER is minimized. Their results show that the STBC can improve the BER of MIMO by (78.6\%). Reference [8] shows that the utilization of MIMO with STBC can enhance the wireless system performance. The authors have investigated the performance of the channel under different combinations of (Tx \& $\mathrm{Rx}$ ) antennas and for different modulation levels. The authors in reference [9] analyzed and presented BER for different fading channels over STBC and spatial multiplexing based MIMO-OFDM system under various modulation techniques. The authors in [10], proposed an approach which is based on powerful combination of pre-coding and equalization, to minimize the BER of the multi-path MIMO channels. Their analysis and simulation results of the proposed design demonstrate that a good advantage can be acquired in terms of channel energy and BER. The authors in [11] have considered the combination between each of the MIMO and OFDM to add support to data rate of the modern communication systems. Also due to the use of the Minimum Mean Square Error (MMSE) with Decision Feedback Decoding (DFB) techniques in the MIMO-OFDM system, their results show a considerable increase in the data rate without considerable difference in BER. In reference [12], two quasi-orthogonal space-time block codes are suggested, one for five and the other for six transmit antennas. These codes have shown full diversity at optimum rotation angles, and they perform better than existing code due to the maximization of the rank of the code word difference matrix. The system efficiency is increased through beamforming 
technique by reference [13]. The authors developed an adaptive antenna system (AAS) to focus the wireless beam between the base station and subscriber station, as a result, $\mathrm{S} / \mathrm{N}$ ratio is enhanced by a gain of $1.8 \mathrm{~dB}$, this satisfied an error rate of $10^{-3}$ in Stanford University Interim channels. The authors in [14] [14] also considered the beamforming technique at the transmit or receive side for the MIMO system over Rician fading channels, their numerical and analytical results aimed to reduce the overhead for estimating channel state information, as a result, this lead to design a more practical massive-MIMO system employing the high gain component based on beamforming scheme. In reference [15] and in order to maximize the received signal to noise ratio, the beamforming or pre-coding vectors are chosen as the singular vectors (corresponding to the largest singular value of the MIMO channel matrix) in single stream MIMO communication systems, In this work, they obtained lower bounds for the received SNR and the corresponding channel capacity, under this mismatched beamforming.

Due to the existence of fading in the wireless communication system, in this paper, MIMO-OFDM with STBC is considered to combat this fading and reduce its effect on the bit error rate and system performance, as well as directivity through beamforming is also investigated to clarify its effect on enhancing spatial diversity and minimizing the co-channel interference effect on the system error rate.

\section{Wireless Communication System Performance}

\subsection{MIMO Channel and H Matrix}

Recently MIMO communications are extensively used to improve the wireless communication system performance, MIMO refers to that both transmitter and receiver consists of multiple antennas. MIMO is also considered the most effective solution for channel fading in high scattering environment, which is caused by multipath, and Figure 5 bellow shows a $2(\mathrm{Tx}) \times 2(\mathrm{Rx})$ MIMO system.

Through the process of sending multiple copies of the data over different antennas, the SNR as well as BER are improved, by taking the advantage of diversity, which means sending multiple copies of signals, then the best one can be chosen and processed at receiver side.

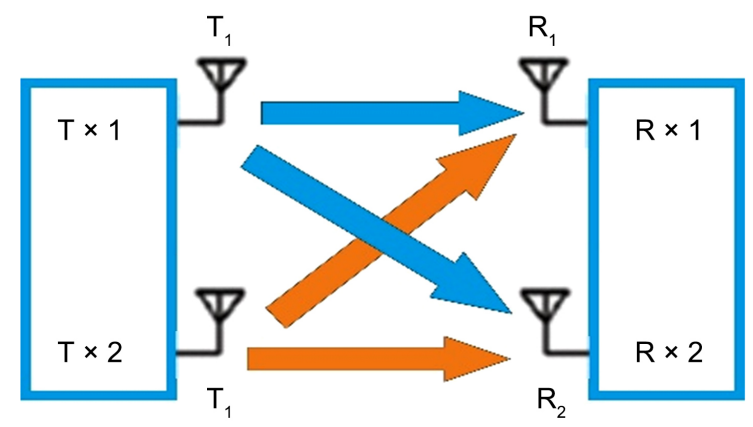

Figure 5. Shows a $2(\mathrm{Tx}) \times 2(\mathrm{Rx})$ MIMO communication system. 
For MIMO system, the communication channel can be presented as a matrix of $\mathrm{Nt} \times \mathrm{Nr}$ dimension (where $\mathrm{Nt}$ and $\mathrm{Nr}$ equal number of transmit and receive antennas respectively). The received ( $r$ ) signal will be a vector of dimension $\mathrm{Nr} \times$ 1 and it can be given as:

$$
\mathrm{r}=\mathrm{Hx}+\mathrm{n}
$$

where $\mathrm{H}$ is the channel matrix and contains $\mathrm{Nt} \times \mathrm{Nr}$ complex elements.

$\mathrm{x}$ is the transmitted vector of dimension $\mathrm{Nt} \times 1$.

$\mathrm{n}$ is the additive noise vector of dimension $\mathrm{Nr} \times 1$ and in matrix form Equation (1) can be written as:

$$
\left[\begin{array}{c}
r_{1} \\
r_{2} \\
\vdots \\
r_{N r}
\end{array}\right]=\left[\begin{array}{cccc}
h_{11} & h_{12} & \cdots & h_{1 N t} \\
h_{21} & h_{22} & \cdots & h_{2 N t} \\
\vdots & \vdots & & \vdots \\
h_{N r 1} & h_{N r 2} & \cdots & h_{N r N t}
\end{array}\right]\left[\begin{array}{c}
x_{1} \\
x_{2} \\
\vdots \\
x_{N t}
\end{array}\right]+\left[\begin{array}{c}
n_{1} \\
n_{2} \\
\vdots \\
n_{N r}
\end{array}\right]
$$

$r_{i}$ is the received signal at ith receiving antenna, $x_{i}$ is the transmitted symbol through the $i$ th transmitting antenna and $h_{i j}$ is the complex channel coefficient of the wireless link between ith receive antenna and th transmit antenna.

Channel modeling is a very important issue to consider for analyzing the performance of wireless communication systems. In most of the situation, it is supposed that the received signal is a collection of many multipath components which are generated due to the reflections caused by the various obstacles between the transmitter and receiver. As a result, both the real and imaginary parts of the channel can be modeled as Gaussian distributed. The magnitude gain of such channels follows Rayleigh distribution, and for that reason, these channels are denoted as Rayleigh fading channels [1].

For MIMO channel, capacity can be calculated as follow:

$$
C_{\text {MIMO }}=\mathrm{BW} * \log _{2}\left(1+N_{t} N_{r} \mathrm{SNR}\right)
$$

where:

$\mathrm{BW}=$ channel bandwidth.

$N_{t}, N_{r}=$ transmission and reception elements, respectively.

$\mathrm{SNR}=$ signal to noise ratio.

\subsection{STBC Performance Analysis}

Space-time block coding is very effective means of achieving transmit diversity, while the other ones of diversity may have limited performance. Such codes can be easily generalized to the case of multiple receiving antennas, as well, this provides receive diversity in addition to transmit diversity. The received set of signals can be decoded efficiently using any simple linear decoding method at the receiver side [16].

To make transmit diversity, the STBC transmission scheme is normally used as a simple way of coding and decoding scheme for MIMO wireless communication systems [1].

According to this coding scheme, a block of incoming bits of data are encoded 
and transmuted across the spatially separated transmitting antennas.

In the STBC systems, the information data is transmitted in blocks (each block has $\mathrm{r}$ symbols, where $r=N_{t}$ ) over time slots (where n time slots are required for one block transmission and $n \geq r$ ). STBC symbol can be mathematically expressed as [1] [7]:

$$
x=\left[\begin{array}{cccc}
s_{11} & s_{12} & \cdots & s_{1 n} \\
S_{21} & s_{22} & \cdots & s_{2 n} \\
\vdots & \vdots & & \vdots \\
S_{r 1} & s_{r 2} & \cdots & s_{r n}
\end{array}\right]
$$

where $s_{i j}$ is one of the symbols or its (transformed version).

For $N_{t} \times N_{r}$ MIMO system over Rayleigh fading channel, the theoretical performance analysis under this coding can be approximated for large signal to noise ratio as [16]:

$$
P_{b}=2\left(\begin{array}{c}
2 N_{t} N_{r}-1 \\
N_{t} N_{r}
\end{array}\right)\left(\frac{N_{t}}{4 \sin ^{2}\left(\frac{\pi}{M}\right)}\right)^{N_{t} N_{r}}\left(\frac{1}{\rho}\right)^{N_{t} N_{r}}
$$

where:

$\mathrm{P}_{\mathrm{b}}=$ probability of bit error rate;

$N_{t}=$ number of Tx antennas;

$N_{r}=$ number of $\mathrm{Rx}$ antennas;

$\rho=$ signal to noise ratio $(\mathrm{SNR})$ in $\mathrm{dB}$;

$M=$ modulation level.

\subsection{Beamforming}

Multiple antenna elements can be employed at the base station transmitters in order to provide directivity for the transmitted signal, hence improving the effective SNR of the channel. As shown in Figure 6 below, base station can focus its transmitted signal toward the desired receiver (mobile A), thus the received signal power can be increased significantly at the intended destination, and dramatically reducing possible interference caused to other users in the system. Also if, multiple antennas are available at the mobile, then mobile A can also effectively obtain a receive antenna pattern to pick up the transmitted signal efficiently, and to reduce the effect of interference it sees.

The basic concept behind directivity or beamforming is the transmission of the signal over each of the multiple antennas, with a certain gain and a phase shift. Different techniques can be used to find suitable coefficients for use. For instance, a direction-of-arrival algorithm can be used, and the coefficients can be computed to make the effective antenna pattern have a main beam in the desired direction. Position information can be obtained, according to the feedback from receiver to the transmitter, this information can also be used to compute the required coefficient for making directivity in the desired direction as well [16]. 


\section{Proposed System Model for MIMO-OFDM Wireless Communication System}

In this paper, the proposed model of the wireless communication system is shown in Figure 7 . In this model, the direct path delay is $(5 \mu \mathrm{sec})$ and the maximum spread delay is $(3.68 \mu \mathrm{sec})$.

To avoid the multipath problem, the OFDM symbol time (Ts) should be (at least) equal to the longest (spread) delay time $\tau \mathrm{d}$ (i.e. $\mathrm{TS} \geq \tau \mathrm{d}$, where $\tau \mathrm{d}$ is the difference between longest path delay and direct path delay). The subcarriers spacing frequency $(\Delta f)$ is the invers of OFDM symbol time $(\Delta f=1 / \mathrm{TS})$. Finally for channel band width of BW, to combat the multipath problem of the proposed communication system, the required minimum number of subcarriers $(\mathrm{N})$ can be calculated as:

$$
N=\mathrm{BW} / \Delta F
$$

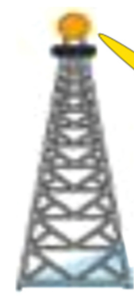

B.S

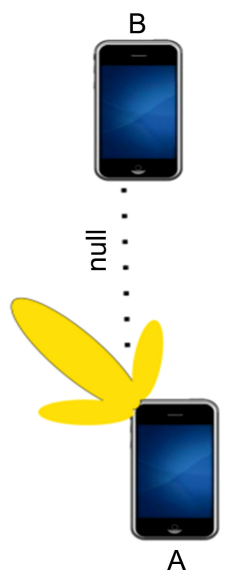

Figure 6. Shows beamforming Principals.

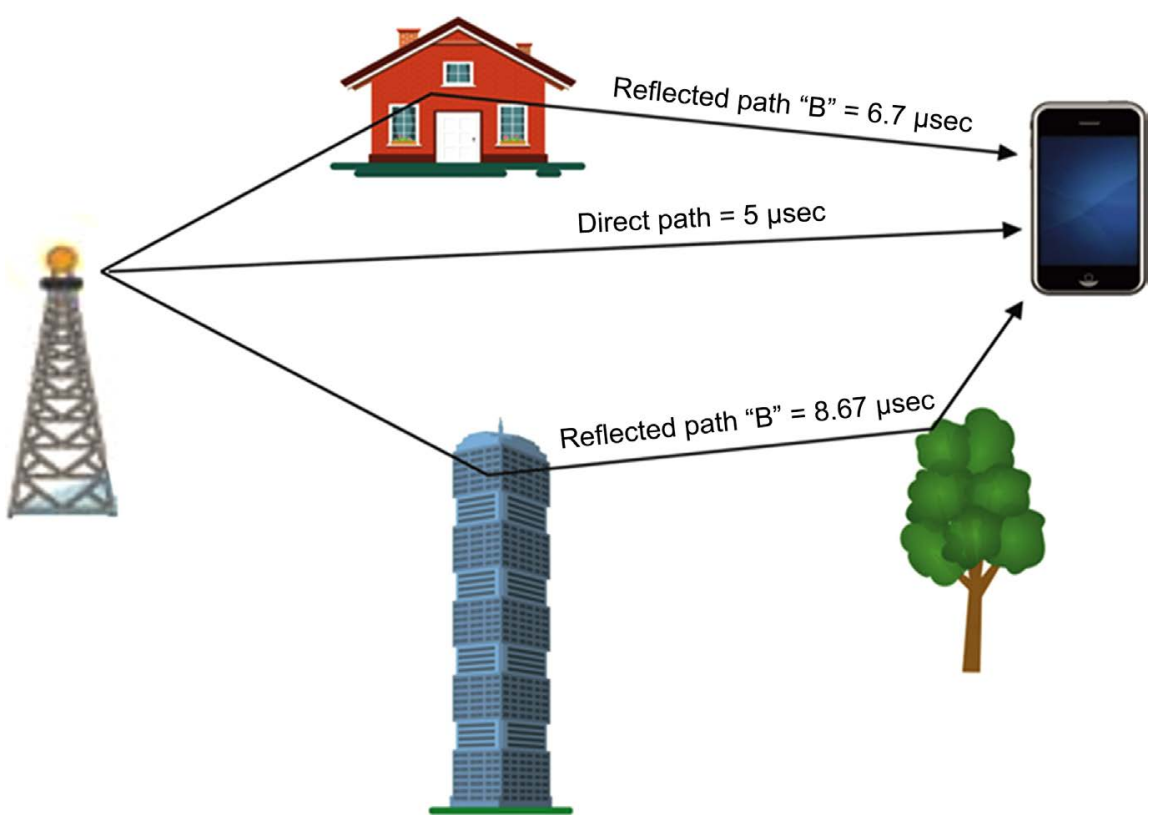

Figure 7. Proposed MIMO-OFDM communication system. 


\subsection{Modeling and Simulation of the Proposed System}

The proposed communication system, is designed, modeled and simulated using MATLAB (Ver. R19). The model consists of base station, cellular mobile, and (when directivity introduced) 6 co-channel interferers are located at different angles and distances in accordance with cellular cluster sizes $(k=4, k=7)$, and their co-channel interferers direction, to show the effect of interferers on transmitted signal.

The area of cell and co-channel interference distance is related to cell radius according to the following formulas:

$$
\begin{aligned}
& A=\frac{3 \sqrt{ } 3}{2} r^{2} \\
& D=r \sqrt{3 k}
\end{aligned}
$$

where: $A=$ area of cell and $r=$ radius of cell.

$D=$ distance to co-channel cell.

$k=$ number of cells in the cluster.

The MIMO-OFDM system, which is designed to combat the multipath and fading problems according to our proposed communication system, is modeled and simulated under the following assumption and simulation parameters as shown in Table 1.

Table 1. Modeled system simulation parameters.

\begin{tabular}{cc}
\hline Modulation & $16-\mathrm{QAM}$ \\
\hline $\mathrm{N}$ (no. of sub-carriers) & 5 \\
Direct path spreading time $(\mu \mathrm{s})$ & 3.67 \\
$\tau \mathrm{d}(\mu \mathrm{s})$ & 20 \\
Channel BW $(\mathrm{MHz})$ & $\mathrm{STBC}$ \\
Encoding & $2,4 \& 8$ \\
No. of Tx elements & $1 \& 2$ \\
No. of Rx elements & 6 \\
No. of interferers & $5,9.5$ \\
Distance between interferers and base station $(\mathrm{Km})$ & variable \\
SNR (dB) & $0,60,120,180,240 \& 300$ \\
Interferers angles (degree) & variable \\
Mobile angle (degree) & 250 \\
Maximum received power from an interferer $(\mathrm{mW})$ & 0.02 \\
\hline \\
\hline
\end{tabular}


- Direct path delay between base station and mobile is $5 \mu \mathrm{s}$.

- The maximum spread delay is $3.67 \mu$ s.

- Maximum cell radios is $1.5 \mathrm{Km}$.

- To be consistent with the co-channel interferers, the maximum number of interferer is 6 .

- Maximum power of base station is $250 \mathrm{~W}$.

- Maximum received interferer power is $0.02 \mathrm{~mW}$.

- When the effect of directivity and co-channel interference on system performance are investigated the following are assumed:

1) $\mathrm{S} / \mathrm{N}$ ratio is constant and for two values ( 5 and $15 \mathrm{~dB}$ ).

2) All the cell's BS are on the same level.

The simulated model directivity for different number of base station transmitting antennas with co-channel interferers position are shown in Figure 8 bellow.

\subsection{Results}

Due to the obstacles between Tx. and $\mathrm{Rx}$, transmitted signal severs from fading as illustrated in the proposed system, to eliminate the effect of this fading and combat multipath problems MIMO-OFDM system is designed and modeled as well as STBC is considered to reduce interference among spatial streams and as a results BER and system reliability are improved. To direct the energy in certain direction beamforming is used and its effect with co-channel interference is also investigated. With respect to the $2 \times 1$ spatial stream, it is known that applying directivities of $4 \times 1$ and $8 \times 1$ streams will introduce gains of $\approx 3 \mathrm{~dB}$ and $6 \mathrm{~dB}$ respectively. The simulation results are given below as follows:

- Without Directivity

The modeled system is simulated under different antennas configuration with and without STBC. The simulation results for these configuration or spatial streams are shown in Figure 9. For all these streams, it is clear as the $\mathrm{S} / \mathrm{N}$ increases
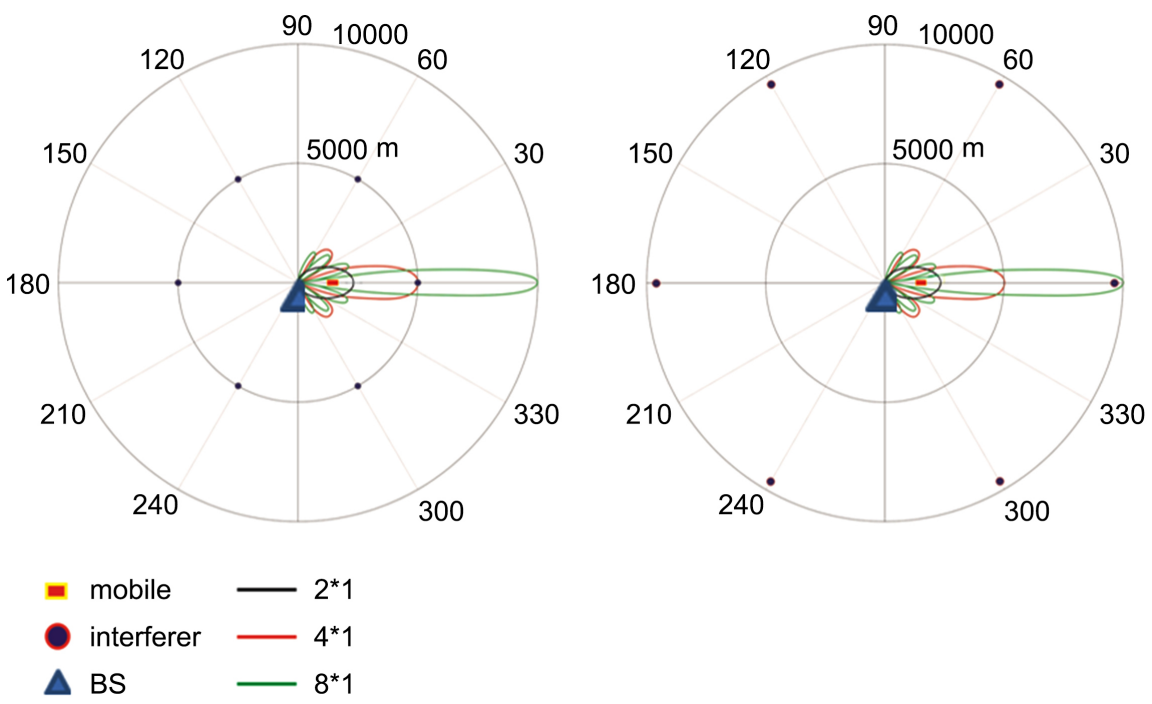

Figure 8. Shows mobile, interferers and BS beamforming. 
the BER decreases and it has the minimum value of $10^{-5}$ for $2 \times 2$ antennas around $25 \mathrm{~dB}$. The figure also shows high consistent between theoretical and simulation results at high $\mathrm{S} / \mathrm{N}$ values, because the mathematical formula (3) is approximated for high $\mathrm{S} / \mathrm{N}$ values. Finally the STBC on average introduces around $1 \mathrm{~dB}$ gain for the different streams.

- With Directivity

As shown in Figure 10 and Figure 11, for the $2 \times 1$ spatial stream at high S/N $(=15 \mathrm{~dB})$ there is more consistent between the theoretical value and simulated value, however at $5 \mathrm{~dB}$ there is a slight difference between the theoretical and simulation value, this is due to the approximation that made on theoretical values where the theoretical formula is approximated for high $\mathrm{S} / \mathrm{N}$ values, as well as there is no effect for the directivity, as long as the $(2 \times 1$ case $)$ is almost slight the omni-directional case.

When the mobile unit is located on the $\mathrm{x}$-axis $\left(\varnothing=0^{\circ}, 1.5 \mathrm{Km}\right.$ from the BS) and directivity is introduced $(4 \times 1,8 \times 1)$, it can be seen from Figure 11 that the highest effect for interferers is at $\varnothing=0^{\circ}$ (or $60^{\circ}, 120^{\circ}, 180^{\circ}, 240^{\circ}$ and $300^{\circ}$ ), where the BER values (for $4 \times 1$ spatial stream) are $8 \times 10^{-3}$ and $2.5 \times 10^{-3}$ and (for $8 \times 1$ spatial stream) are $7 \times 10^{-3}$ and $2 \times 10^{-3}$ at interferers positions $5 \mathrm{Km}$ and $9.5 \mathrm{Km}$ from $\mathrm{BS}$ respectively. As the mobile unit is made to move around the $\mathrm{BS}$ on a circle with $1.5 \mathrm{Km}$ radius, the effect of interferers on the directivity BER performance start to decrease as long as the BS main beam will follow and directed toward the mobile unit. At $\varnothing \approx 15^{\circ}$ (where the first null is occurred) the BER is noticeably decreased, then it shows slight increase at $\emptyset \approx 20^{\circ}$ due to the first side lobe effect. The minimum effect of interferers is noticed at $\varnothing=30^{\circ}$ and the BER is minimum values of $3 \times 10^{-4}$ and $1 \times 10^{-4}$ at 5 and $9.5 \mathrm{Km}$ interferers distances respectively.

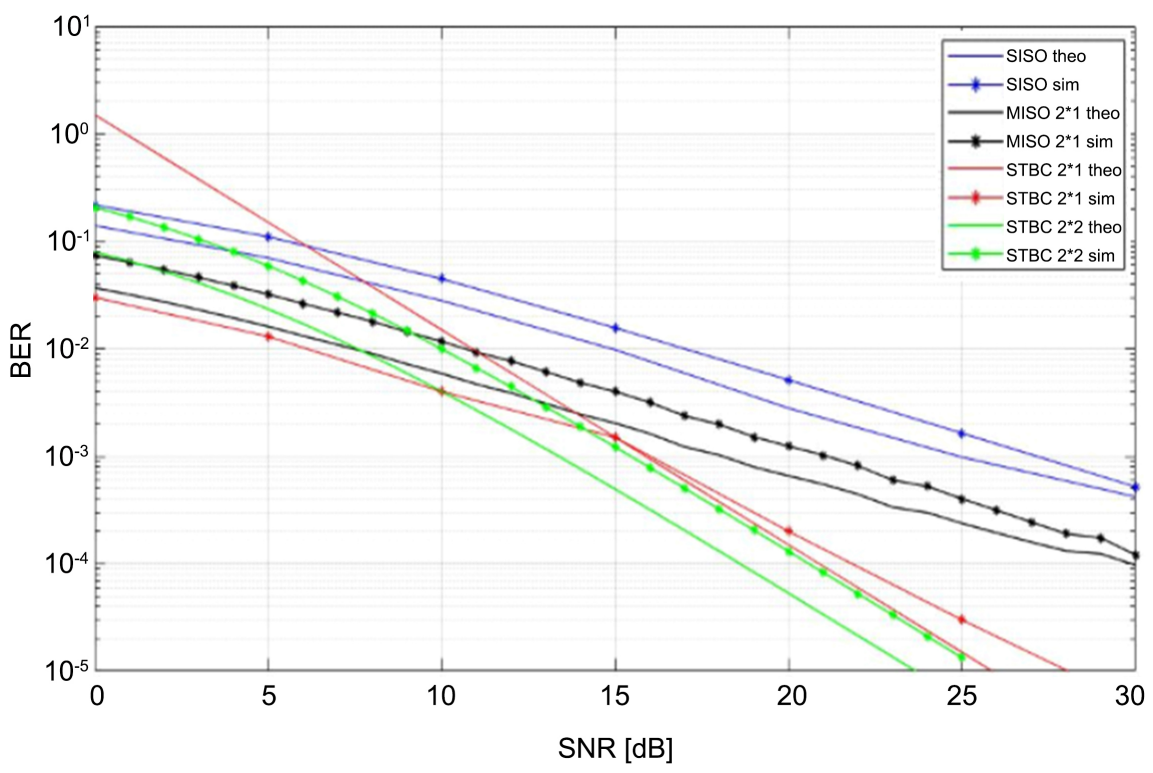

Figure 9. Shows theoretical and simulation performance for different MIMO antenna configurations with STBC. 


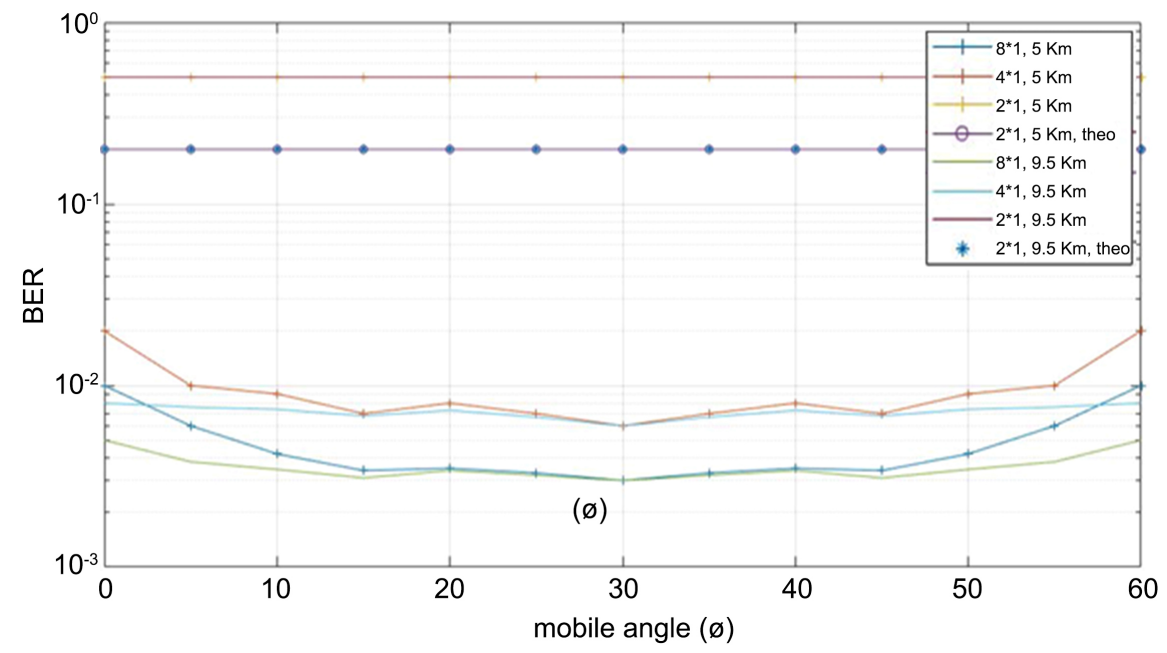

Figure 10. BER vs. mobile angles for 6 interferers at distance of $5 \& 9.5 \mathrm{Km}$ with $5 \mathrm{~dB}$ SNR.

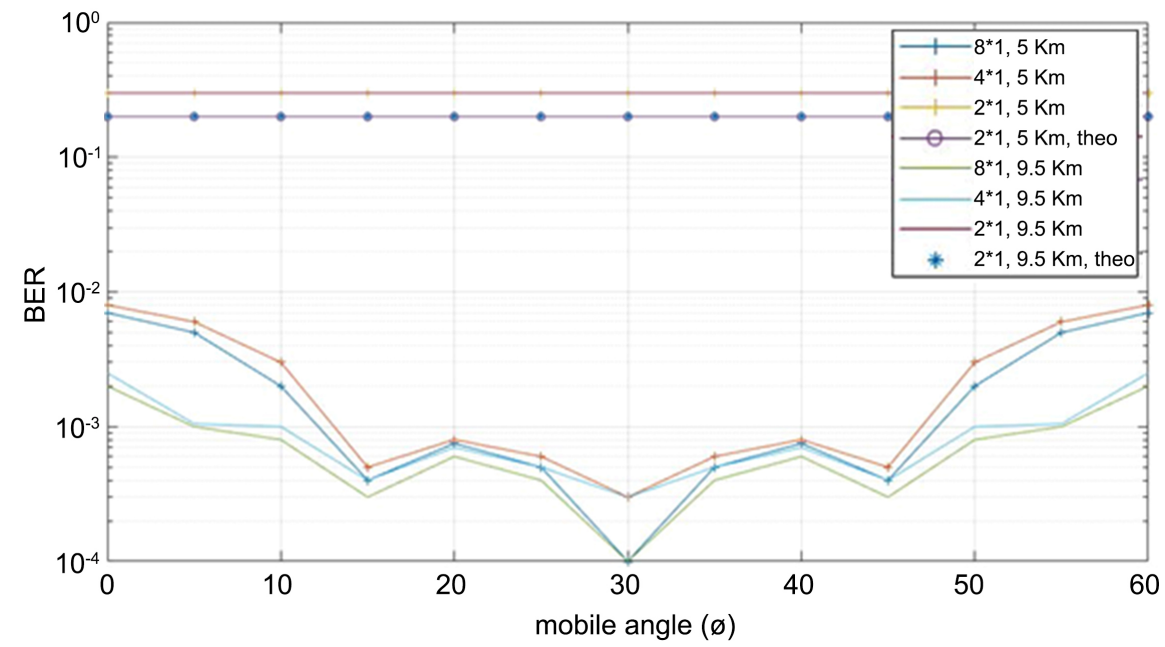

Figure 11. BER vs. mobile angles for 6 interferers at distance of $5 \& 9.5 \mathrm{Km}$ with $15 \mathrm{~dB}$ SNR.

In comparison with no directivity case $(2 \times 1$, where the BER vary from $2 \times$ $10^{-2}$ to $2 \times 10^{-3}$ at 5 and $15 \mathrm{~dB}$ respectively), it's obvious when directivity is introduced, then at $\varnothing \approx 30^{\circ}$ the BER at $5 \mathrm{~dB}$ S/N (Figure 10) can be decreased up to $6 \times 10^{-3}$ (at $5 \mathrm{Km}$ interferers) and $4 \times 10^{-3}$ (at $9.5 \mathrm{Km}$ interferers), while in 15 $\mathrm{dB}$ S/N (Figure 11) can be decreased up to $3 \times 10^{-4}$ and $1 \times 10^{-4}$ for interferers positions $5 \mathrm{Km}$ and $9.5 \mathrm{Km}$ from BS respectively. So at mobile angle $\varnothing=30^{\circ}$ the best BER improvement values at $15 \mathrm{~dB}$ S/N are $85 \%$ and $95 \%$, while at $5 \mathrm{~dB} S / \mathrm{N}$ the improvement values are $70 \%$ and $80 \%$ for $5 \mathrm{Km}$ and $9.5 \mathrm{Km}$ respectively.

The interesting thing is that there is a degradation in the BER system performance when the mobile unit $\left(\varnothing=0^{\circ}, 60^{\circ} \ldots\right)$ and the interferes are on the same line (i.e. the interferer is on the main lobe of $\mathrm{BS}$ ). This degradation is more obvious at $15 \mathrm{~dB} \mathrm{~S} / \mathrm{N}$ and when interferers are close to $\mathrm{BS}(5 \mathrm{Km})$, the BER increased for $2 \times 10^{-3}$ to $8 \times 10^{-3}$ with $30 \%$ degradation. 
Table 2. Shows BER for different spatial streams at $\mathrm{S} / \mathrm{N}=5 \mathrm{~dB}$.

\begin{tabular}{ccccc}
\hline No. & Distance $(\mathrm{Km})$ & BER $(2 \times 1)$ & BER $(4 \times 1)$ & BER $(8 \times 1)$ \\
\hline 1 & 5 & $5 \times 10^{-1}$ & $2 \times 10^{-2}$ & $1 \times 10^{-2}$ \\
2 & 9.5 & $5 \times 10^{-1}$ & $8 \times 10^{-3}$ & $5 \times 10^{-3}$ \\
\hline
\end{tabular}

Table 3. Shows BER for different spatial streams at $\mathrm{S} / \mathrm{N}=15 \mathrm{~dB}$.

\begin{tabular}{ccccc}
\hline No. & Distance $(\mathrm{Km})$ & BER $(2 \times 1)$ & BER $(4 \times 1)$ & BER $(8 \times 1)$ \\
\hline 1 & 5 & $3 \times 10^{-1}$ & $8 \times 10^{-3}$ & $7 \times 10^{-3}$ \\
2 & 9.5 & $3 \times 10^{-1}$ & $2.5 \times 10^{-3}$ & $2 \times 10^{-3}$ \\
\hline
\end{tabular}

Table 2 and Table 3 summarize the highest BER values (at $\varnothing=0$ ) for the above system.

\section{Conclusion}

This paper investigates the effect of MIMO-OFDM and STBC on BER for the cellular systems, and how SNR can be improved when applying beamforming over such wireless communication systems. Extensive simulation processes accomplished to optimize the network performance. At transmitter side, three scenarios of $(2,4 \& 8)$ antennas were investigated to show the effect of multiple antennas with beamforming on system performance. The results show that using STBC with directivity of $4 \times 1$ and $8 \times 1$ antenna streams provide gains in S/N around $4 \mathrm{~dB}$ and $7 \mathrm{~dB}$ respectively. As a result of these gains, maximum improvements of $85 \%$ and $95 \%$ in BER value are obtained for large cluster size (9.5 $\mathrm{Km}$ ), however (when the mobile unit and co-channel interfere are on the same angle $\left(0^{\circ}, 60^{\circ}, 120^{\circ}, 180^{\circ}, 240^{\circ} \& 300^{\circ}\right)$, a degradation of $30 \%$ is noticed for small cluster size $(5 \mathrm{Km})$. As future works directivity can be applied in vertical or elevation plane as well through the use of two-dimensional arrays and investigate its effect in reducing the co-channel interference for small size cluster.

\section{Acknowledgements}

I gratefully acknowledge the support and generosity for my supervisor, without which the present study could not have been completed. Thanks also go to Dr. Mohammed Alhabbar and Dr. Yousif Awad for their assistance in references collection.

\section{Conflicts of Interest}

The authors declare no conflicts of interest regarding the publication of this paper.

\section{References}

[1] Kumbhani, B. and Kshetrimayum, R.S. (2017) MIMO Wireless Communications 
over Generalized Fading Channels. CRC Press, Boca Raton. https://doi.org/10.1201/9781315116778

[2] Hampton, J.R. (2019) Introduction to MIMO Communications. Cambridge University Press, New York.

[3] Sim, Z.A., Reine, R., Zang, Z.Q. and Gopal, L. (2017) PAPR and BER Reduction in MU-MIMO-OFDM Systems via a Set of Waveforms. MIMO Proc. of the 2017 IEEE International Conference on Signal and Image Processing Applications, Malaysia, 12-14 September 2017, 55-60.

[4] Tang, W.W. and Kang, S.L. (2020) Design of MIMO-PDMA in 5G Mobile Communication System. IET Communications, 14, 76-83.

https://doi.org/10.1049/iet-com.2018.5837

[5] Farej, Z.K. and Jasim, M.M. (2018) Investigation on the Performance of the IEE802.11n Based Wireless Networks for Multimedia Services. 2nd International Conference for Engineering, Technology and Sciences of Al-Kitab, Karkuk, 4-6 December 2018, 48-53.

[6] Jeiad, H.A. and Al-Bahadili, R.J.S. (2019) Multipath Fading Effects on Uncoded and Coded Multiple Frequency Shift Keying Performance in Mobile Wireless Communications. Journal of Engineering and Sustainable Development, 23, 18-33.

https://doi.org/10.31272/jeasd.23.4.2

[7] Thaher, R.H. and Radhy, H.Y. (2015) MIMO Channel Capacity Improvement Using Space Time Coding. Journal of Engineering and Development, 19, 1-15.

[8] Bharti, P.K. and Rawat, P. (2018) Alamouti-STBC Based Performance Estimation of Multi Tx and Rx Antenna over MIMO-OFDM. Proceedings of the 2 nd International Conference on Trends in Electronics and Informatics (ICOEI 2018), Tirunelveli, 11-12 May 2018, 1277-1281. https://doi.org/10.1109/ICOEI.2018.8553804

[9] Ravibabu, T. and Dharma Raj, C. (2018) BER Analysis of Spatial Multiplexing and STBC MIMO-OFDM System. 4th International Conference on Devices, Circuits and Systems (ICDCS), Coimbatore, 6-17 March 2018, 110-116.

[10] Doanh, B.Q., Quan, D.T., et al. (2019) An Efficient Design of Precoding and Equalization to Reduce BER of Multi-Path MIMO Channels. 3 rd International Conference on Recent Advances in Signal Processing, Telecommunications \& Computing, Hanoi, 21-22 March 2019, 15-19.

[11] Abdul-Latef, W.M. and Mawjoud, S.A. (2008) Performance Improvement of Cellular Systems Using MIMO-OFDM. AL-Rafidain Engineering Journal (ARE), 16, 69-79.

[12] Sindhu, P. and Hameed, A. (2015) Efficient Quasi-Orthogonal Space-Time Block Codes for Five and Six Transmit Antennas. IEEE International Conference on Electronics, Computing and Communication Technologies (CONECCT), Bangalore, 10-11 July 2015, 1-5.

[13] Kadhim, M.A. (2014) Design and Implementation of Adaptive Antenna System in a New LTE 3GPP Transceivers Based Multiwavelet Signals. Iraqi Journal of Applied Physics, 10, 11-16.

[14] Zhang, Y., Yue, D.-W. and Jia, Y.N. (2015) Further Results on the Beamforming Transmission Scheme Only Using the Specular Component for Massive-MIMO Systems. Fifth International Conference on Instrumentation and Measurement, Computer, Communication and Control (IMCCC), Qinhuangdao, 18-20 September 2015, 997-1001.

[15] Myers, N.J. and Kannu, A.P. (2016) Impact of Channel Estimation Errors on Single 
Stream MIMO Beamforming. IEEE Communications Letters, 21, 1345-1348.

[16] Duman, T.M. and Ghrayeb, A. (2007) Coding for MIMO Communication Sytems. Wiley, Hoboken. https://doi.org/10.1002/9780470724347 